\title{
Estimation of lithium-ion battery state-of-charge using an extended kalman filter
}

\author{
Mouhssine Lagraoui ${ }^{1}$, Ali Nejmi ${ }^{2}$, Hassan Rayhane ${ }^{3}$, Abderrahim Taouni ${ }^{4}$ \\ ${ }^{1,2,3}$ Laboratory of Mathematics and Physics, Faculty of Sciences and Technics, Sultan Moulay Slimane University, \\ BeniMellal, Morocco \\ ${ }^{4}$ Electrical Engineering Department, Mohammadia School of Engineers, Mohammed V University in Rabat, Morocco
}

\begin{tabular}{l} 
Article Info \\
\hline Article history: \\
Received Oct 27, 2020 \\
Revised Feb 19, 2021 \\
Accepted May 28, 2021 \\
\hline
\end{tabular}

\section{Keywords:}

Battery management system

Battery state of charge

Extended kalman filter

Lithium-ion battery

\begin{abstract}
The main goal of a battery management system (BMS) is to estimate parameters descriptive of the battery pack operating conditions in real-time. One of the most critical aspects of BMS systems is estimating the battery's state of charge (SOC). However, in the case of a lithium-ion battery, it is not easy to provide an accurate estimate of the state of charge. In the present paper we propose a mechanism based on an extended kalman filter (EKF) to improve the state-of-charge estimation accuracy on lithium-ion cells. The paper covers the cell modeling and the system parameters identification requirements, the experimental tests, and results analysis. We first established a mathematical model representing the dynamics of a cell. We adopted a model that comprehends terms that describe the dynamic parameters like SOC, open-circuit voltage, transfer resistance, ohmic loss, diffusion capacitance, and resistance. Then, we performed the appropriate battery discharge tests to identify the parameters of the model. Finally, the EKF filter applied to the cell test data has shown high precision in SOC estimation, even in a noisy system.
\end{abstract}

This is an open access article under the CC BY-SA license.

\section{Corresponding Author:}

Ali Nejmi

Electrical Department

Faculty of Sciences and Technics

Sultan My Slimane University, BP 523, 23000, Beni Mellal, Morocco

Email: alinejmi@yahoo.fr

\section{INTRODUCTION}

Batteries have become the essential limiting factor for several applications, including portable electronic devices and electric/hybrid vehicles, to mention but a few. Furthermore, Batteries have shown many drawbacks in various aspects. They have smaller energy and power densities compared to fossil fuels, their lifetime is uncertain, and their behavior and characteristics are changing over time [1], [2]. In recent years, lithium-ion batteries (Li-ion) have risen as the preferred technology for portable devices and transportation applications because of their excellent chemical composition, good lifetime, and better efficiency compared to other battery types. However, a Li-ion battery requires a battery management system (BMS) assuring power control and general enhanced stability. A battery management system is mandatory to maintain optimal battery performance. The key part of a BMS is that it permits the battery state of charge (SOC) estimation in real-time; hence, the available capacity can be determined. With the estimated SOC, BMS can optimize battery energy efficiency, predict the state variables, and protect batteries from overcharging and over-discharging. We define the SOC as the amount of energy left in a battery compared to the initial energy when it was fully charged. "Obviously, from a practical point of view, we cannot measure SOC directly according to its definition without varying it dramatically" [3]. 
Multiple studies have proposed many different SOC estimation methods. The simplest and most used method is coulomb counting (counting amp-hours) [4], [5]. Nevertheless, this strategy is based on an open-loop algorithm that has the disadvantage of a significant measurement error accumulation due, especially to uncertain disturbances and the difficulty of accurately determining the SOC initial value [3]. The open-circuit battery voltage (OCV) measurement method is also used. However, it requires a sufficient rest period to ensure a stable battery voltage.

A couple of new methods based on neural networks and fuzzy logic [6]-[9] were discussed in the literature. They depend on intelligence-based and computational algorithms, involve many training sample data, and are rarely realized in real-time applications. Hybrid simplified kalman filter [10], linear kalman filter (KF), extended kalman filter (EKF) [11]-[18], and the unscented kalman filter [19]-[21] were also proposed. The state of charge (SOC) model-free tuning method is mainly used in the hybrid electric vehicle system. "The methodology focuses on the variable SOC target governed by a linear equation of the actual vehicle speed" [22]. The KF is a recursive algorithm for SOC estimation. It has the advantage of assuring an optimal estimate of the state of charge even in the presence of noisy disturbances. Besides, KF based methods do not require accurate knowledge of the SOC initial value [23]. EKF algorithm is used not only for the battery model parameters identification but also for SOC estimation in real-time operation [17], [24]. It is primarily suitable for dynamic systems. Moreover, good precision in battery SOC estimate depends heavily on the battery model precision, parameters initial values, and predetermined variables, even in case of system noise such as covariance matrix relevance and average value.

In this paper, a lithium-ion battery (nominal capacity of $1.8 \mathrm{Ah}$ ) is selected as the object of our research investigation. EKF is applied to our Thevenin model to estimate SOC. It allows the SOC value to be continuously corrected and adjusted in real-time. Based on the analysis of many experiments on SOC (as detailed in the following sections), one could conclude that EKF produces a good and accurate estimate of SOC, even for a noisy system.

\section{BATTERY MODELING AND PARAMETERS IDENTIFICATION}

Different modeling methods are mentioned in several papers. Recently, equivalent circuits and electrochemical models are the most cited and most widely used in battery modeling. So far, many models based on various assumptions have been proposed for the dynamic modelling of lead-acid batteries. The following works can be referred to:- Battery modeling using the Thevenin model [12], where the temperature is assumed to be constant; - "Dynamic modeling of afuel cell by Bruno Francois [25]. In the later model, all parameters are supposed to be constant; - Nonlinear time series models using neural network systems have also been used to enhance the existing nonlinear dynamic models" [26].

A SOC estimation process is based on a model that replicates internal battery state variables such as open-circuit voltage (OCV) and SOC, among other parameters. The lithium-ion battery system's SOC prediction model is developed using support vector machine modeling (SVM) [27]. The cell is considered as a nonlinear dynamic system that can be represented by a state-space as:

$$
\left\{\begin{array}{c}
\dot{X}=F(X, U)+W \\
Y=G(X, U)+V
\end{array}\right.
$$

"Where $\mathrm{X}$ is the state vector defining the system states. U represents the current $\mathrm{I}$, and $\mathrm{Y}$ is the output of the measuring system. $\mathrm{W}$ is the unmeasured "process noise" that affects the state of the system. $\mathrm{V}$ is the measurement noise not affecting the state of the system but results in the output. Moreover, $\mathrm{W}$ and $\mathrm{V}$ are assumed to be Gaussian white noise with zero mean and zero covariance. $F(X, U)$ and $G(X, U)$ are functions specified by the model" [28].

The equivalent circuit model for a lithium battery [18] is shown in Figure $1 . \mathrm{V}_{\text {ocv }}$ is the open-circuit voltage; the surface capacitance $C_{1}$ represents battery diffusion effects. The resistors $R_{\text {in }}$ and $R_{1}$ are (respectively) the internal resistance and transfer resistance. $\mathrm{V}_{1}$ is the voltage across the surface capacitor; $\mathrm{V}_{\mathrm{T}}$ and I are, respectively, the battery terminal voltage and current. From Figure 1, the battery terminal voltage can be written as :

$$
V_{T}=V_{o c v}(S O C)-V_{1}-R_{\text {in }} I
$$

Experimental discharge results on the Li-ion battery see Figure 2 helped define a relationship between the open-circuit voltage and battery charge (OCV-SOC). In fact, this function plays a central role in terms of accuracy in estimating the load state. As a result, we can say that this relationship is the added value 
of the method developed in our current work. The SOC $\left(\mathrm{V}_{\mathrm{ocv}}\right)$ curve is built from the experimental discharge results carried out on the Li-ion battery see Figure 2 . The $\mathrm{SOC}$ is related to $\mathrm{V}_{\text {ocv }}$ as:

$$
V_{o c v}=k_{1}+k_{2} \times S O C+k_{3} \times S O C^{3}
$$

With $k_{1}=3.56, k_{2}=0.356$ and $k_{3}=0.2827$

In this regard, it should be noted that the values of the above parameters depend on the chosen cell.

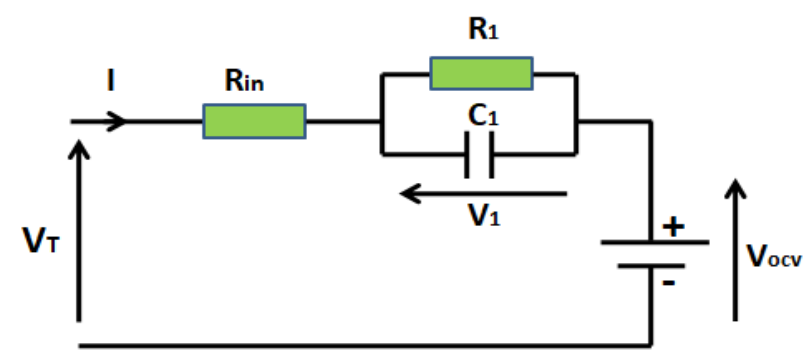

Figure 1. Thevenin model of the Li-ion battery

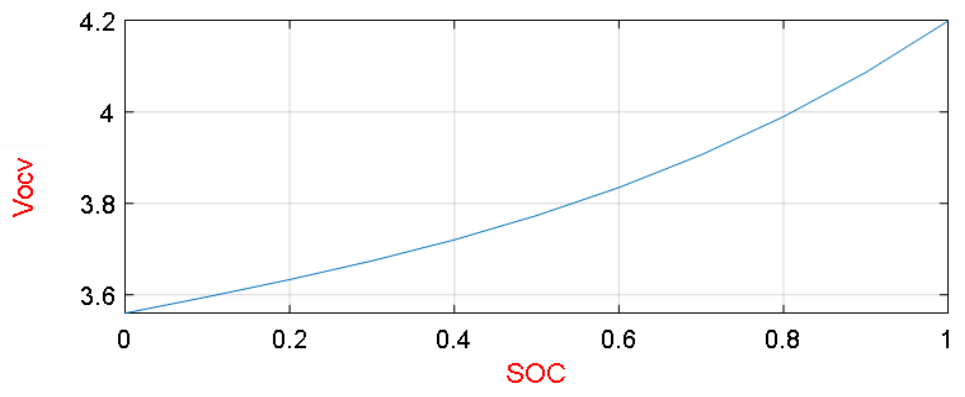

Figure 2. SOC- $\mathrm{V}_{\text {ocv }}$ curve

After a rest period, $\mathrm{V}_{\text {ocv }}$ can be easily measured while the battery is in an open-circuit state. Therefore, SOC can be estimated using (3). However, this case is not always feasible, especially in real-time operation. In effect, while the battery is connected to the system, it will be very difficult to obtain information on SOC since the battery's internal impedance can't be measured during the discharge process (presence of the current I). Therefore, To make the SOC estimate easier, the open-circuit voltage must also be measured in real-time conditions. (as illustrated in (3)). In (4) represents the voltage $\mathrm{V}_{1}$ across the capacitor $\mathrm{C}_{1}$ :

$$
V_{1}=R_{1} \times\left(I-C_{1} \dot{V}_{1}\right.
$$

One of the most critical pieces of information in a BMS is the state of charge, a cell's internal state [14] that we cannot explicitly measure during battery operation. As a result, estimating the SOC value is the only option.

$$
S O C_{\text {batt }}=S O C_{\text {initial }}-\frac{1}{3600 \times A h} \int I d t
$$

where: I: the battery terminal current

Ah:the battery capacity in A.h

$\mathrm{SOC}_{\text {initial: }}$ the initial state of charge

$\mathrm{SOC}_{\text {batt }}$ : the battery SOC

The model parameters are extracted from the Lithium-ion battery voltage response, resulting from the constant current pulse discharge test. Figure 3 and Figure 4 illustrate cell voltage response and current pulse, respectively. 


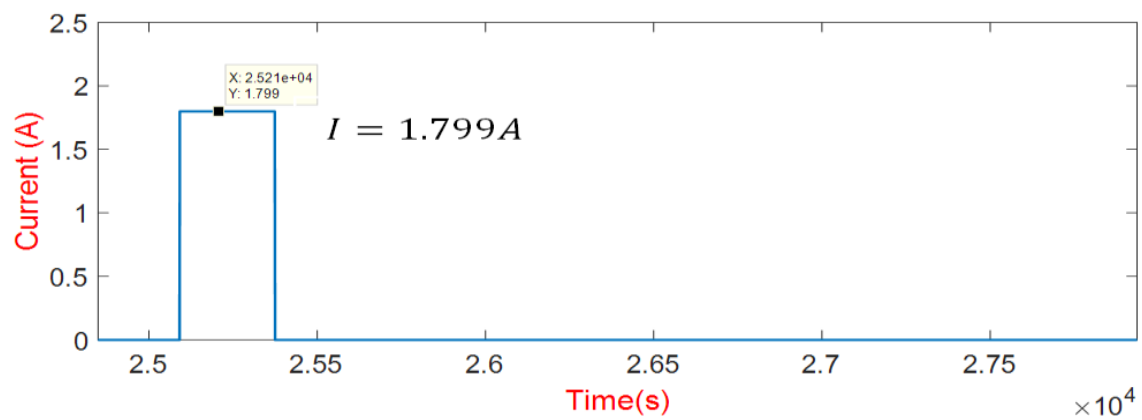

Figure 3. 1C constant current discharge (Lithium-ion battery)

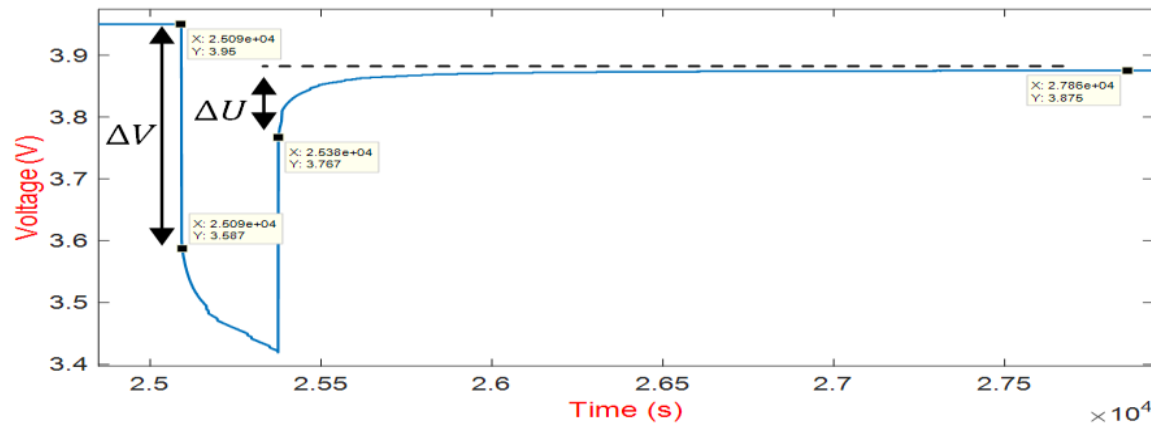

Figure 4. 1C constant current discharge test (Li-ion battery): The voltage response

When the discharge pulse is applied, the voltage response during the battery discharge test, as shown in Figure 4, shows that there is an instantaneous voltage drop $(\Delta V)$. On the other hand, there is an instantaneous increase in voltage once the discharge stops. The tension response proves the existence of internal resistance $R_{\text {in }}$ that can be computed in (6).

$$
R_{\text {in }}=\frac{\Delta V}{I}=\frac{3.95-3.587}{1.799}=0.201 \Omega
$$

The cell diffusion resistance $\mathrm{R}_{1}$ is determined on the voltage curve from the initial instant of the immediate rise in voltage and the moment it reaches a pseudo-stable value during the established regime. We divide $\Delta U$ voltage by discharge test constant current $(\mathrm{I}=1.799 \mathrm{~A})$. Accordingly, $\mathrm{R}_{1}$ is determined as:

$$
R_{1}=\frac{\Delta U}{I}=\frac{3.875-3.767}{1.799}=0.06 \Omega
$$

The ratio of the battery model time constant to the diffusion resistance is used to determine the diffusion capacity $\mathrm{C}_{1}$. From the Figure 4 , the $\mathrm{C}_{1}$ discharge time constant $\tau$ is determined as:

$$
\tau=R_{1} C_{1}=25436-25380=56 s
$$

$\tau$ is the time required to reach $(1-1 / \mathrm{e}) \approx 63.2 \%$ of $\Delta U$ see Figure 4 . Therefore, the diffusion capacity is:

$$
C_{1}=\frac{\tau}{R_{1}}=\frac{56}{0.06}=933 F
$$

The Table 1 shows the battery model parameters:

Table 1. Battery model parameters

\begin{tabular}{cccc}
\hline Model parameter & $\mathrm{R}_{\text {in }}$ & $\mathrm{R}_{1}$ & $\mathrm{C}_{1}$ \\
\hline Value & $0.201 \Omega$ & $0.06 \Omega$ & $933 \mathrm{~F}$ \\
\hline
\end{tabular}


$\mathrm{R}_{1}$ and $\mathrm{C}_{1}$ are dynamic parameters, but for the sake of simplicity, they have been kept constant in the following, as their variation is not significant during battery charging and discharging. To validate the calculated parameters, we compared the voltage measured value and the estimated one, as illustrated in Figure 5. The discharge tests performed on the cell included a series of constant current discharge pulses followed by rest intervals. The current profile of the experiment is shown in Figure 5 (a). We can see, according to Figure $5(\mathrm{~b}-\mathrm{c})$, that the estimated voltage curve perfectly follows the measured voltage one with a small maximum error of $0.04 \mathrm{~V}$. These results confirm that the estimated SOC curve converges towards the real one. This validates the accurateness of the adopted model.

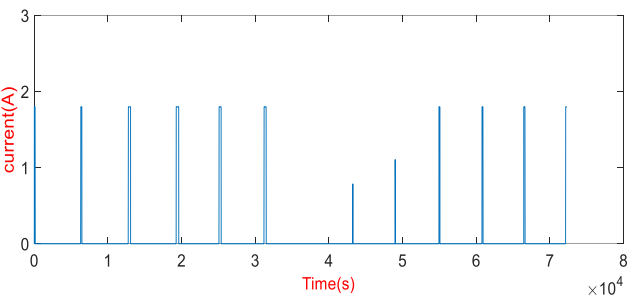

(a)

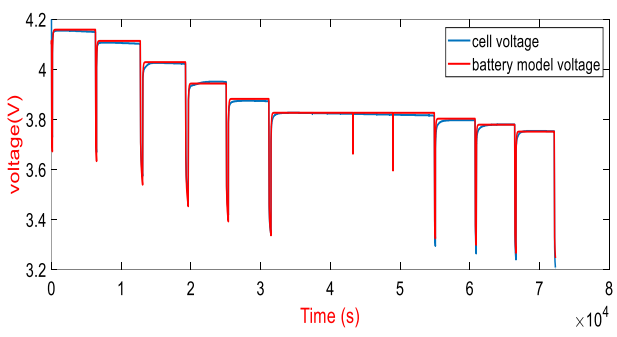

(b)

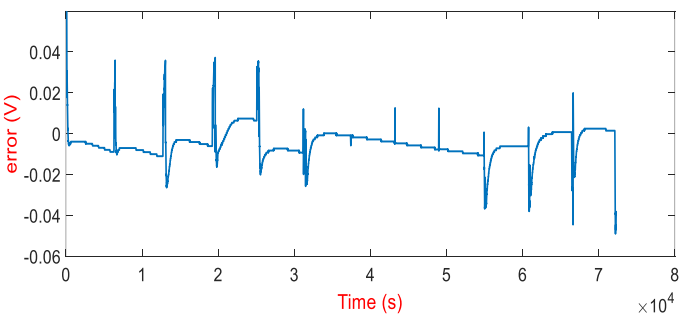

(c)

Figure 5. These figures are, (a) test current, (b) step charge curve, (c) the battery voltage estimation error

\section{USING EXTENDED KALMAN FILTER FOR SOC ESTIMATION}

The kalman filter is the most widely used method in tracking technologies. It is also applied as an estimation algorithm in linear systems. Under the designation of EKF, it has been adapted for nonlinear systems [29]. The EKF is especially used for prediction in dynamic systems, like tracking, navigation, and global positioning. The EKF has recently entered the battery field. The kalman filter concept is based on recursive mathematical equations that allow the repetition of calculations at every step. The system's output relies on the prior input so that the state vector depends on the current output as a function of its input.

Moreover, the battery is intrinsically a nonlinear system because of its dynamic behavior, on the one hand, and the current and voltage sensors, on the other. In the battery model, the voltage is the output and the current is the input. Each sampling period's state is calculated and assessed by an observer. Furthermore, the observer is tuned to track all system parameters. In this regard, a specific state variable represents a parameter. Battery systems are inherently nonlinear. Therefore, in this chapter, the EKF, with a linearization process, is investigated. The equations system represents our nonlinear system:

$$
\begin{aligned}
& y_{k}=g\left(x_{k}, u_{k}\right)+v \\
& x_{k+1}=f\left(x_{k}, u_{k}\right)+w
\end{aligned}
$$

$f\left(x_{k}, u_{k}\right)$ and $g\left(x_{k}, u_{k}\right)$ functions are linearized using a first-order Taylor series expansion around the point $x_{k}=\hat{x}_{k-1}$ for the process model, and $x_{k}=\hat{x}_{k}^{-}$for the measurement model. We assume that both functions are differentiable at all operating points, as shown in (12)-(13).

$$
g\left(x_{k}, u_{k}\right) \approx g\left(\hat{x}_{k}^{-}, u_{k}\right)+\left.\frac{\partial g\left(x_{k}, u_{k}\right)}{\partial x_{k}}\right|_{x_{k}=\hat{x}_{k}^{-}}\left(x_{k}-\hat{x}_{k}^{-}\right)
$$




$$
f\left(x_{k}, u_{k}\right) \approx f\left(\hat{x}_{k-1}, u_{k}\right)+\left.\frac{\partial f\left(x_{k}, u_{k}\right)}{\partial x_{k}}\right|_{x_{k}=\hat{x}_{k-1}}\left(x_{k}-\hat{x}_{k-1}\right)
$$

By substituting (12) and (13), in (10) and (11), the linearized process and measurement models become:

$$
\begin{aligned}
& g\left(x_{k}, u_{k}\right) \approx \hat{G}_{k} x_{k}+g\left(\hat{x}_{k}^{-}, u_{k}\right)-\hat{G}_{k} \hat{x}_{k}^{-}+v \\
& x_{k+1} \approx \hat{A}_{k} x_{k}+f\left(\hat{x}_{k-1}, u_{k}\right)-\hat{A}_{k} \hat{x}_{k-1}+w
\end{aligned}
$$

We define $\hat{A}_{k}$ and $\widehat{G}_{k}$ as:

$$
\begin{aligned}
& \hat{A}_{k}=\left.\frac{\partial f\left(x_{k}, u_{k}\right)}{\partial x_{k}}\right|_{x_{k}=\hat{x}_{k-1}} \\
& \hat{G}_{k}=\left.\frac{\partial g\left(x_{k}, u_{k}\right)}{\partial x_{k}}\right|_{x_{k}=\hat{x}_{k}^{-}}
\end{aligned}
$$

Recursive steps of the extended kalman filter algorithm can be summarized as:

a. Initialize the original parameters.

$$
\begin{aligned}
& \hat{x}_{k-1}=E\left(x_{0}\right) \\
& P_{k-1}=E\left[\left(x_{0}-\hat{x}_{0}\right)\left(x_{0}-\hat{x}_{0}\right)^{T}\right]
\end{aligned}
$$

b. Estimate the predicted state

$$
\hat{x}_{k}^{-}=f\left(\hat{x}_{k-1}, u_{k}\right)
$$

c. Update estimate covariance

$$
P_{k}^{-}=\hat{A}_{k} P_{k-1} \hat{A}_{k}^{T}+Q
$$

d. Determine near-optimal kalman gain

$$
K_{k}=P_{k}^{-} \widehat{G}_{k}^{T}\left(\widehat{G}_{k} P_{k}^{-} \hat{G}_{k}^{T}+R\right)^{-1}
$$

e. Update state estimate

$$
\hat{x}_{k}=\hat{x}_{k}^{-}+K_{k}\left(y_{k}-g\left(\hat{x}_{k}^{-}, u_{k}\right)\right)
$$

f. Predict estimate covariance

$$
P_{k}=\left(I-K_{k} \widehat{G}_{k}\right) P_{k}^{-}
$$

g. Repeat the recursive filter calculation from step 2 to step 6

The discrete extended kalman estimator for the lithium-ion cell is derived from the previously constructed model. The states used to model the behavior of the battery are:

$$
\begin{aligned}
& x_{1}=S O C ; \quad x_{2}=V_{1} \\
& \begin{aligned}
x & =\left[\begin{array}{ll}
x_{1} & x_{2}
\end{array}\right]^{T} \\
& =\left[\begin{array}{ll}
S O C & V_{1}
\end{array}\right]^{T}
\end{aligned}
\end{aligned}
$$

The battery current is the process control variable $u=I$.

Both the current integration process expression and the Thevenin model mathematical relations are discretized and linearized to define the state transition as: 


$$
\begin{aligned}
& f(x, u)=\left[f_{1} f_{2}\right]^{T} \\
& f_{1}=S O C_{k-1}-I_{k-1} \frac{\Delta t}{3600 \cdot A H}=x_{1}-u \frac{\Delta t}{3600 \cdot A H} \\
& f_{2}=V_{1 \mid k-1} \cdot e^{-\frac{\Delta t}{\tau}}-I_{k-1} R_{1}\left(1-e^{-\frac{\Delta t}{\tau}}\right) \\
& \quad=x_{2} \cdot e^{-\frac{\Delta t}{\tau}}-u \cdot R_{1}\left(1-e^{-\frac{\Delta t}{\tau}}\right)
\end{aligned}
$$

With $\tau=R_{1} C_{1} \quad$ and $\quad \Delta t$ : sampling period.

The measured (observable) parameters are limited to the cell terminals voltage. Thus, the measurement vector is $y=V_{T}$. The observation is given:

$$
\begin{aligned}
g(x, u) & =k_{1}+k_{2} \times S O C+k_{3} \times S O C^{3}-V_{1}-R_{1} \times I \\
& =k_{1}+k_{2} \times x_{1}+k_{3} \times x_{1}{ }^{3}-x_{2}-R_{1} \times u
\end{aligned}
$$

We must determine the two Jacobians in order to use this model in the EKF. As a result, the state transition equations' derivative with respect to state variables is:

$$
\hat{A}_{k}=\left.\frac{\partial f\left(x_{k}, u_{k}\right)}{\partial x_{k}}\right|_{x_{k}=\hat{x}_{k-1}}=\left(\begin{array}{ll}
F_{11} & F_{12} \\
F_{21} & F_{22}
\end{array}\right)
$$

After calculating, we get:

$$
F_{11}=1, F_{12}=0, \quad F_{21}=0 \text { and } F_{22}=\mathrm{e}^{-\frac{\Delta \mathrm{t}}{\tau}}
$$

According to the state variables, the observation derivative is:

$$
\widehat{G}_{k}=\left.\frac{\partial g\left(x_{k}, u_{k}\right)}{\partial x_{k}}\right|_{x_{k}=\hat{x}_{k}^{-}}=\left[\begin{array}{ll}
G_{1} & G_{2}
\end{array}\right]
$$

After computing, we obtain:

$$
\begin{aligned}
& G_{1}=k_{2}+3 k_{3} x_{1}^{2} \\
& G_{2}=-1
\end{aligned}
$$

\section{EXPERIMENTAL RESULTS}

Figure 6 illustrates the test bench used for discharge tests. It includes a 1.8Ah Li-ion cell and a programmable DC electronic loads. Discharge tests are carried out on a fully charged battery cell. The aim is to compare the experimental results of SOC estimate with the reference values. Figure 7 (a-b) shows results for the case in which the SOC estimate and its reference have the same initial value, while Figure 8 (a-b) represents the results for different initial values.

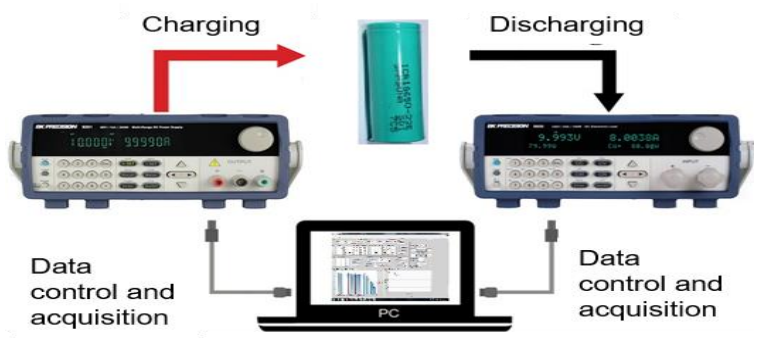

Figure 6. The schematic of the test bench 
Results detailed below show that the SOC estimate has a maximum error of $1 \%$. This error is inherent to modeling errors. The results show that the calculated cell model parameters produce high accuracy in open-circuit voltage estimation. While the function between $\mathrm{V}_{\mathrm{OCV}}$ and SOC leads to good accuracy in SOC estimation. We notice, as well, that the extended kalman filter observer algorithm quickly converges to the SOC reference with high accuracy, despite the fact that the reference initial value differs from SOC's initial value.

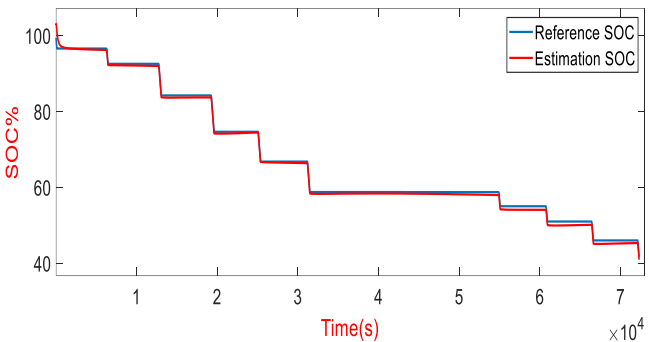

(a)

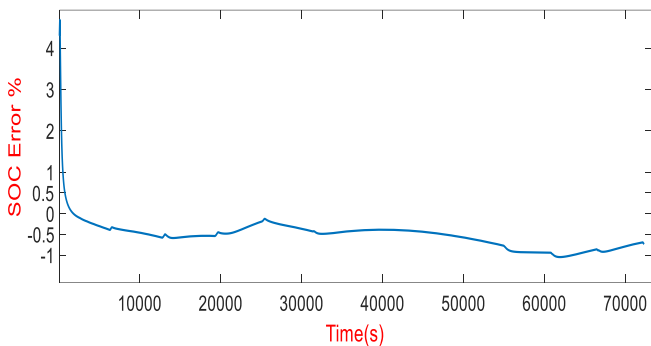

(b)

Figure 7. These figures are; (a) SOC estimate results, (b) SOC error when the initial SOC is known

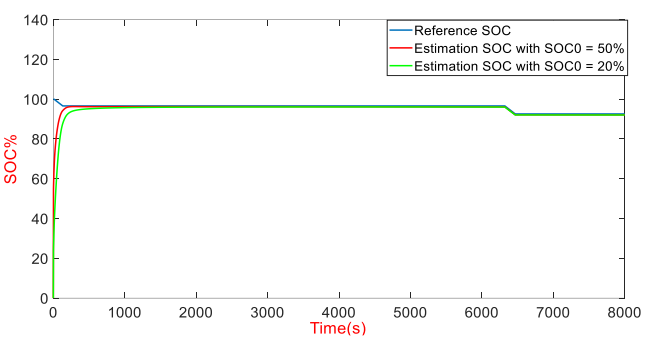

(a)

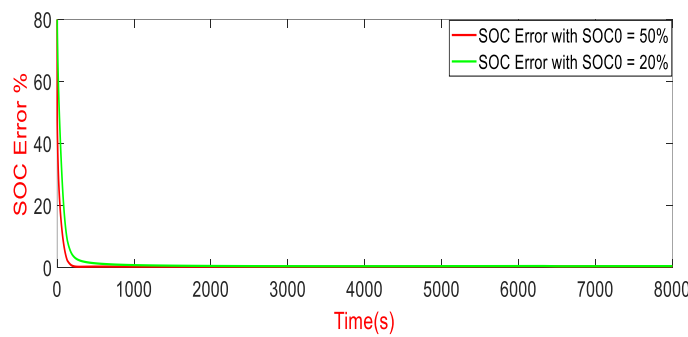

(b)

Figure 8. These figures are; (a) SOC estimate results, (b) SOC error when the initial SOC is unknown

\section{CONCLUSION}

In the present paper, we determined the main three parameters of the battery Thevenin model (ohmic resistance, polarization resistance, polarization capacitance) through experimental discharge tests. Moreover, tests were conducted to establish a connection between the cell open-circuit voltage and the stateof-charge. This relationship plays a key role in accurately estimating the state of charge of the battery. This relationship varies from cell to cell, depending on the cell's constituent materials. Then, an EKF algorithm based on the Thevenin model was established. EKF performances were assessed by carrying out constant current discharge experimental tests. According to the comparison analysis between experimental results and reference values, EKF based on the Thevenin model has shown a good accuracy performance in SOC estimate. Furthermore, the results met the precision and rapid convergence requirements in SOC estimate, with an error of less than $1 \%$, independently of the initial value. SOC estimation combined with a real-time parameters identification approach for the dynamic battery model will be the subject of future research.

\section{REFERENCES}

[1] M. Lagraoui, S. Doubabi, A. Rachid, "SOC estimation of Lithium-ion battery using Kalman filter and Luenberger observer: A comparative study,” 2014 International Renewable and Sustainable Energy Conference (IRSEC), 2014, pp. 636-641, doi: 10.1109/IRSEC.2014.7059849.

[2] D. Zhang, Y. Ma, Q. -W. Bai, "Estimation of Lithium-ion battery state of charge," Proceedings of the 30th Chinese Control Conference, 2011, pp. 6256-6260.

[3] R. Restaino, W. Zamboni, "Comparing particle filter and extended kalman filter for battery State-Of-Charge estimation," IECON 2012 - 38th Annual Conference on IEEE Industrial Electronics Society, 2012, pp. 4018-4023, doi: 10.1109/IECON.2012.6389247. 
[4] Ng K. S, Moo C. S, Chen Y. P, Hsieh Y. C, "Enhanced coulomb counting method for estimating state-of-charge and state-of-health of lithium-ion batteries," Appl Energy, vol. 86, no. 9, pp. 1506-1511, 2009. https://doi.org/10.1016/j.apenergy.2008.11.021.

[5] C. T. Lin, Q. S. Chen, J. P. Wang, W. H. Huang, Y. C. Wang, "Improved Ah counting method for state of charge estimation of electric vehicle batteries," Tsinghua University Science Technology, vol. 46-2, pp. 247-251, 2006.

[6] B. Cheng, Z. Bai, Cao Binggang, "State of charge estimation based on evolutionary neural network," Energy Conversion and Management, vol. 49, no. 10, pp. 2788-2794, 2008. https://doi.org/10.1016/j.enconman.2008.03.013.

[7] C. Piao, W. Fu, Jin-Wang, Z. Huang, C. Cho, "Estimation of the state of charge of Ni-MH battery pack based on artificial neural network," INTELEC 2009 - 31st International Telecommunications Energy Conference, 2009, pp. 1-4, doi: 10.1109/INTLEC.2009.5351908.

[8] Hossain Lipu, M. S. Hannan, M. A. Hussain A, Saad M. H. M, "Optimal BP neural network algorithm for state of charge estimation of lithium-ion battery using PSO with PCA feature selection," Journal of Renewable and Sustainable Energy, vol. 9, no. 6, pp. 1-17, 2017. doi:10.1063/1.5008491.

[9] Z. Li, D. Liu, F. Lu, X. Heng, Y. Guo, Q. Jiang, "Research on SOC estimation of lithium battery based on GWOBP neural network," 2020 15th IEEE Conference on Industrial Electronics and Applications (ICIEA), 2020, pp. 506-510, doi: 10.1109/ICIEA48937.2020.9248364

[10] F. Albu, L. T. T. Tran, S. Nordholm, "The Hybrid Simplified Kalman Filter for Adaptive Feedback Cancellation," International Conference on Communications (COMM), 2018, pp. 45-50, doi: 10.1109/ICComm.2018.8484823.

[11] M. Souaihia, B. Belmadani, R. Taleb, "A robust state of charge estimation for multiple models of lead acid battery using adaptive extended Kalman filter," Bulletin of Electrical Engineering and Informatics, vol. 9, no. 1, pp. 1-11, 2020, doi: 10.11591/eei.v9i1.1486.

[12] H. He, R. Xiong, X. Zhang, F. Sun, J. Fan, "State-of-Charge Estimation of the Lithium-Ion Battery Using an Adaptive Extended Kalman Filter Based on an Improved Thevenin Model," in IEEE Transactions on Vehicular Technology, vol. 60, no. 4, pp. 1461-1469, May 2011, doi: 10.1109/TVT.2011.2132812.

[13] J. Lee, O. Nam, B. H. Cho, "Li-ion battery SOC estimation method based on the reduced order extended Kalman filtering," Journal of Power Sources, vol. 174, no. 1, pp. 9-15, 2007.

[14] L. Zhi, Z. Peng, W. Zhifu, S. Qiang, R. Yinana, "State of charge estimation for Li-ion battery based on extended Kalman filter," Energy Procedia, vol, 105, pp. 3515-3520, 2017, https://doi.org/10.1016/j.egypro.2017.03.806.

[15] G. L. Plett, "Extended Kalman filtering for battery management systems of LiPB-based HEV battery packs: Part 1. Background," Journal of Power Sources, vol. 134, no. 2, pp. 252-261, 2004, https://doi.org/10.1016/j.jpowsour.2004.02.031.

[16] G. L. Plett, "Extended Kalman filtering for battery management systems of LiPB-based HEV battery packs: Part 2. Modeling and identification," Journal of Power Sources, vol. 134, no. 2, pp. 262-276, 2004, https://doi.org/10.1016/j.jpowsour.2004.02.032.

[17] G. L. Plett, "Extended Kalman filtering for battery management systems of LiPB-based HEV battery packs: Part 3. State and parameter estimation," Journal of Power Sources, vol. 134, no. 2, pp. 277-292, 2004. https://doi.org/10.1016/j.jpowsour.2004.02.033

[18] Yu Ding-xuan, Gao Yan-xia, "SOC estimation of Lithium-ion battery based on Kalman filter algorithm," Proceedings of the 2nd International Conference on Computer Science and Electronics Engineering ICCSEE, 2013, pp. -1852-1855, https://doi.org/10.4028/www.scientific.net/AMM.347-350.1852.

[19] C. Piao, Z. Sun, Z. Liang, C. Cho, "SOC Estimation of Lead-Acid Batteries Based on UKF," 2010 International Conference on Electrical and Control Engineering, 2010, pp. 1968-1972, doi: 10.1109/iCECE.2010.484.

[20] S. Santhanagopalan, R. E. White, "State of charge estimation usingan unscented filter for high power lithium ion cells," International Journal Energy Research, vol. 34, no. 2, pp. 152-163, 2009, https://doi.org/10.1002/er.1655.

[21] F. Zhang, G. Liu, L. Fang, "Battery state estimation using Unscented Kalman Filter," 2009 IEEE International Conference on Robotics and Automation, 2009, pp. 1863-1868, doi: 10.1109/ROBOT.2009.5152745.

[22] Ahmad, M. A, Baba. I, Azuma S. I, Sugie T, "Model free tuning of variable state of charge target of hybrid electric vehicles," IFAC Proceedings Volumes, vol. 46, no. 21, pp. 789-793, 2013. https://doi.org/10.3182/20130904-4-JP2042.00066.

[23] O. Barbarisi, F. Vasca, L. Glielmo, "State of charge Kalman filter estimator for automotive batteries," Journal of Control Engineering Practice, vol. 14, no. 3, pp. 267-275, 2006, https://doi.org/10.1016/j.conengprac.2005.03.027.

[24] Q.-H. Meng, Y. C. Sun, Z. L. Cao, "Adaptive extended Kalman filter (AEKF)-based mobile robot localization using sonar," Robotica, vol. 18, pp. 459-473, 2000.

[25] Francois B, Hissel D, Iqbal T, "Dynamic modelling of a fuel cell and wind turbine DC-linked power system," Electrimacs Conference, 2005, pp. 1-6.

[26] El Mehdi Laadissi, Anas El Filali, Malika Zazi, "A Nonlinear TSNN Based Model of a Lead Acid Battery", Bulletin of Electrical Engineering and Informatics, vol. 7, no. 2, pp. 169-175, 2018. doi: 10.11591/eei.v7i2.675.

[27] R. Li et al, "State of Charge Prediction Algorithm of Lithium-Ion Battery Based on PSO-SVR Cross Validation," in IEEE Access, vol. 8, pp. 10234-10242, 2020, doi: 10.1109/ACCESS.2020.2964852.

[28] R. Xiong, H. He, F. Sun, K. Zhao, "Evaluation on State of Charge Estimation of Batteries With Adaptive Extended Kalman Filter by Experiment Approach," in IEEE Transactions on Vehicular Technology, vol. 62, no. 1, pp. 108117, Jan. 2013, doi: 10.1109/TVT.2012.2222684.

[29] Fei Zhang, Guangjun Liu, Lijin Fang, "A battery State of Charge estimation method with extended Kalman filter," 2008 IEEE/ASME International Conference on Advanced Intelligent Mechatronics, 2008, pp. 1008-1013, doi: 10.1109/AIM.2008.4601799. 


\section{BIOGRAPHIES OF AUTHORS}
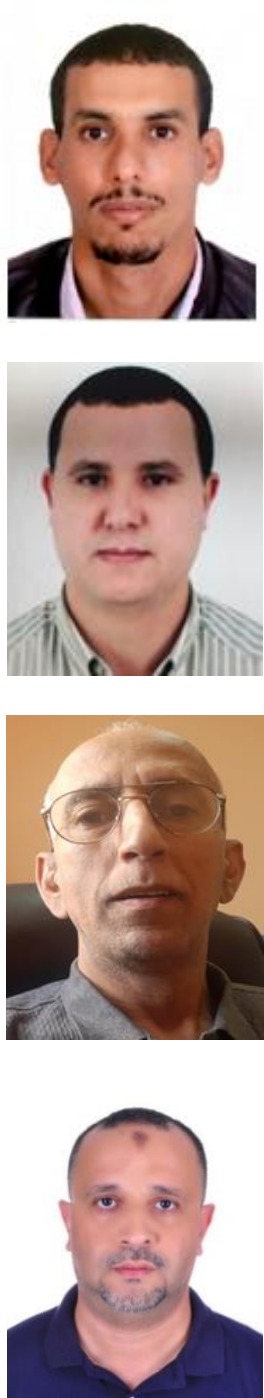

Mouhssine Lagraoui received his diploma in Electrical Engineer from National School of Applied Sciences ENSA Marrakech, Morocco, in 2012. He is currently a postgraduate student pursuing his doctorate's degree under the supervision of Dr Ali Nejmi in Laboratory of Mathematics and Physics, Faculty of Sciences and Technics, Beni Mellal, Morocco. His research concerns with battery management system (BMS).

Ali Nejmi received his diploma in Electrical Engineer and Advanced Graduate Studies degree from Mohammadia School of Engineers, Morocco, in 1995 and 2001, respectively. He received his doctorate degree from Sultan Moulay Slimane University, Morocco, in 2015. He has been an associate professor at the Electrical Department, Faculty of Sciences and Technics, Sultan Moulay Slimane University, Morocco, since 1996. His field of research interest includes nonlinear control of electrical machines, renewable energies plants integration in electrical grids, and hybrid micro-grids.

Hassan Rayhane received his engineer degree in Electrical Engineering from Ecole Nationale Supérieure d'Electricité et de Mécanique (ENSEM) of Casablanca in 1990. He received Postgraduate Diploma (DEA) in Robotic from Laboratory of Robotic of Paris in 1991. He received his PHD diploma in Applied Science from National Institute Polytechnic of Grenoble (INPG) in 2004. He has been an associate professor at the Electrical Department, Faculty of Sciences and Technics, Sultan Moulay Slimane University, Morocco, since 1996. His field of research interest includes nonlinear control of electrical machines and Robotic.

Abderrahim Taouni received the Engineer degree in electrical Engineering from High Institute of Technical Education (ENSET) of Mohammedia in 1997, and the Aggregation in Electrical Engineering from the Ecole Normal Superior of Technical Education (ENSET), Rabat, in 2008. He received the Master degree in ATSII (Automatic, Signal Processing and Industrial Computing) from Faculty of Science and Technology Hassan I university Settat, MOROCCO in 2011. Currently He is Research Professor Laboratory of Industrial engineering, information processing and logistics (GITIL)-Department of Physics, Aïn Chock Science faculty- Hassan II University Casablanca, MOROCCO. His research interests include control strategies for AC machine Drives, renewable energy and batteries. 